\title{
ARTICLE Circulating PACAP peptide and PAC1R genotype as possible transdiagnostic biomarkers for anxiety disorders in women: a preliminary study
}

Rachel A. Ross ${ }^{1,2,3,4,5}$, Susanne S. Hoeppner iD $^{4,5}$, Samantha N. Hellberg ${ }^{40,6}$, Emily B. O'Day ${ }^{4,7}$, Peter L. Rosencrans ${ }^{4,8}$, Kerry J. Ressler ${ }^{3,5,9}$, Víctor May ${ }^{10}$ and Naomi M. Simon ${ }^{4,11}$

Pituitary adenylate cyclase activating polypeptide (PACAP, gene Adcyap1) is a neuropeptide and hormone thought to play a critical role in stress response (Stroth et al., Ann NY Acad Sci 1220:49-59, 2011; Hashimoto et al., Curr Pharm Des 17:985-989, 2011). Research in humans implicates PACAP as a useful biomarker for the severity of psychiatric symptoms in response to psychological stressors, and work in rodent models suggests that PACAP manipulation exerts downstream effects on peripheral hormones and behaviors linked to the stress response, providing a potential therapeutic target. Prior work has also suggested a potential sex difference in PACAP effects due to differential estrogen regulation of this pathway. Therefore, we examined serum PACAP and associated PAC1R genotype in a cohort of males and females with a primary diagnosis of generalized anxiety disorder (GAD) and nonpsychiatric controls. We found that, while circulating hormone levels were not associated with a GAD diagnosis overall ( $p=$ $0.19, g=0.25)$, PACAP may be associated with GAD in females $(p=0.04, g=0.33)$. Additionally, among patients with GAD, the risk genotype identified in the PTSD literature ( $\mathrm{rs} 2267735, \mathrm{CC}$ genotype) was associated with higher somatic anxiety symptom severity in females but lower somatic anxiety symptom severity in males $(-3.27,95 \% \mathrm{Cl}[-5.76,-0.77]$, adjusted $p=0.03)$. Taken together, the associations between the risk genotype, circulating PACAP, and somatic anxiety severity were stronger among females than males. These results indicate a potential underlying biological etiology for sex differences in stress-related anxiety disorders that warrants further study.

Neuropsychopharmacology (2020) 45:1125-1133; https://doi.org/10.1038/s41386-020-0604-4

\section{INTRODUCTION}

Pituitary adenylate cyclase activating polypeptide (PACAP, gene Adcyap1) is a neuropeptide and hormone thought to play a critical role in stress response both peripherally and in the brain [1, 2]. Prior work in humans indicates it may be a useful biomarker for the severity of psychiatric symptoms in response to psychological stressors, and work in animal models points to the potential utility of PACAP as a therapeutic target. Many studies have implicated PACAP and its receptor, PAC1R, in the regulation of the hypothalamic pituitary adrenal (HPA) axis. In fact, PACAP is densely expressed in both the central nervous system nuclei that regulate the HPA axis (e.g. paraventricular hypothalamus), as well as the peripheral endocrine organs (both pituitary and adrenal glands) [3-7]. Studies report that the highest concentration of PACAP is found in hypothalamic regions compared to other regions in the rodent $[3,6]$ and human [5] brain, where it is thought to act as a glutamatergic neuromodulator at the postsynaptic neuron [8]. Moreover, PACAP has been shown to modulate the release of corticotrophin releasing factor (CRF) from the hypothalamus, and downstream circulating corticosterone in rodents. These represent key markers of the stress response and indicate one potential mechanism for the effect of central PACAP in the stress axis [9-11].

Similarly, preclinical studies suggest that whole-body PACAP manipulation exerts downstream effects on peripheral hormones and behaviors linked to the stress response. For example, wholebody PACAP- and PAC1R-deficient mice show reduced corticosterone and decreased anxiety-like behavior in response to a chronic stressor, suggesting that both peripheral and central PACAP are required for normal stress responding [11-16]. Interestingly, chronic stress appears to promote increased PACAP levels and to induce similar anxiety-like behaviors, as supported by increased transcript expression of ADCYAP1 and ADCYAP1R1 [17-19]. Together, these findings suggest that the PACAPergic system may serve as an important regulator of the stress response, and may contribute to the pathogenesis and maintenance of stress-related pathology.

Consequently, a number of groups have investigated the relationship between PACAP and stress-related conditions in

\footnotetext{
${ }^{1}$ Department of Psychiatry, Beth Israel Deaconess Medical Center, Boston, MA, USA; ${ }^{2}$ Department of Medicine, Division of Endocrinology, Beth Israel Deaconess Medical Center, Boston, MA, USA; ${ }^{3}$ Department of Psychiatry, McLean Hospital, Belmont, MA, USA; ${ }^{4}$ Department of Psychiatry, Massachusetts General Hospital, Boston, MA, USA; ${ }^{5}$ Department of Psychiatry, Harvard Medical School, Boston, MA, USA; ${ }^{6}$ Department of Psychology \& Neuroscience, University of North Carolina at Chapel Hill, Chapel Hill, NC, USA; ${ }^{7}$ Department of Psychology, Temple University, Philadelphia, PA, USA; ${ }^{8}$ Department of Psychology, University of Washington, Seattle, WA, USA; ${ }^{9}$ Department of Psychiatry and Behavioral

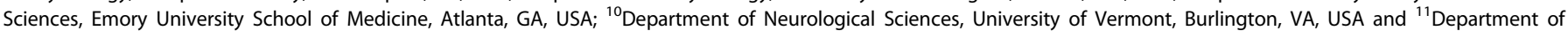
Psychiatry, NYU Grossman School of Medicine, New York, NY, USA

Correspondence: Rachel A. Ross (rross4@partners.org)
}

Received: 17 July 2019 Revised: 30 November 2019 Accepted: 30 December 2019

Published online: 7 January 2020 


\section{6}

humans characterized by HPA axis dysregulation and aberrant fear and memory processes, such as posttraumatic stress disorder (PTSD) [20-28]. Among the few studies conducted to date, findings have been mixed with some reports demonstrating significant associations between the CC genotype of ADCYAP1R1 (rs2267735) and PTSD in traumatized women but not men $[25,27,28]$. Additionally, PTSD symptom severity and diagnostic status has been positively correlated with the circulating long form of PACAP (PACAP38) [28]. Thus, human studies also indicate a link between whole-body PACAP and stress response. Chronic stress appears to be an important trigger for these psychological and behavioral manifestations of PACAP dysfunction [24, 25, 27].

Given this evidence and the breadth of literature supporting the role of lifetime stress and maladaptive autonomic responses in anxiety disorders [29], it is possible that PACAP may confer risk for anxiety and maintain anxiety-related pathology more broadly. Generalized anxiety disorder (GAD) is a chronic anxiety condition characterized by persistent, excessive worry as well as heightened somatic and autonomic symptoms, including muscle tension or aches, increased arousal, sweating, irritability, fatigue, and concentration and sleep difficulties for at least 6 months $[30,31]$. Further, the lifetime prevalence of GAD is higher among females $(7.7 \%)$ than males (4.6\%) [32]. While GAD is not linked to specific life events like PTSD and trauma, there is evidence it is heightened by environmental stressors, perhaps with gene -environment interactions similar to those suggested by a postdisaster study of an NPY gene (NPY rs16147) and GAD [33]. Only one study to date, however, has examined the potential role of PACAP in GAD maintenance, demonstrating that ADCYAP1R1 genotypes A/A and A/G (SNP rs2856966) were associated with enhanced treatment response to venlafaxine $X R$. Individuals with genotype $\mathrm{A} / \mathrm{G}$ of $A D C Y A P 1 R 1 \mathrm{rs} 2856966$ demonstrated the most robust response to treatment, including greater reductions in clinical symptoms and higher rates of remission, in a sample $(N=$ 156) of treatment-seeking men and women with GAD [34]. To our knowledge, no studies have investigated the association of circulating PACAP or the genotype of its receptor PAC1R with GAD diagnosis or symptom severity. However, a few studies have implicated a role for PACAP in regulation of central symptoms that are components of the diagnostic criteria for GAD, such as sleep dysregulation and somatic symptoms $[31,35,36]$.

In rodents, PACAP has been shown to influence neuronal areas that regulate rapid eye movement (REM) sleep and circadian rhythms, such as the pontine reticular nucleus (PnO) and suprachiasmatic nucleus (SCN) [8], and PAC1R is highly distributed in the SCN [37-39]. Furthermore, in animals, central and peripheral PACAP manipulations have been shown to alter sympathetic nerve activity, breathing, heart rate, and headache [40-45]. Thus, PACAP system dysfunction in humans may lead to both the somatic symptoms and sleep-related difficulties commonly experienced by patients with GAD, and this gap in knowledge about the involvement of PACAP in GAD led to the studies described below.

In this paper, we present the findings of two studies: (1) an examination of circulating PACAP levels in adults with a primary diagnosis of GAD compared to a sample of participants without any psychiatric diagnosis; and (2) an investigation of PACAP levels and anxiety and stress symptoms among different PAC1R SNP genotypes in a sample of adults with a primary diagnosis of GAD. Based on prior research linking chronic stress with activation of the PACAP system, we hypothesized that circulating PACAP would be higher in people with GAD after controlling for effects of sex and other potential confounders. We further hypothesized that among patients with a primary diagnosis of GAD, the risk genotype previously identified in the PTSD literature (rs2267735, CC genotype) would be associated with differences in circulating PACAP as well as anxiety symptom severity and poor sleep quality. Additionally, we expected that the association between GAD and circulating PACAP, as well as the association between the risk genotype and circulating PACAP, anxiety severity, and sleep quality, would be stronger among females.

\section{MATERIALS AND METHODS}

\section{Participants}

Patients with a primary diagnosis of GAD and participants without any psychiatric diagnosis (healthy controls, $\mathrm{HC}$ ) were enrolled in a larger ancillary study at the Center for Anxiety and Traumatic Stress Disorders at the Massachusetts General Hospital between 2003 and 2017 [46-48]. Eligible participants were willing and able to sign informed consent and over the age of 18. Individuals were not eligible if they presented with a lifetime history of psychotic disorders, bipolar disorder, mental disorder due to a medical condition or substance, or met criteria for an alcohol or substance abuse or dependence disorder or eating disorders in the past 6 months. Pregnancy or lactation, unstable medical conditions, and acute suicidal risk were also exclusionary. In addition to these criteria, $\mathrm{HCs}$ were excluded if they met the criteria for an affective or anxiety disorder, as determined by MINI or SCID in the past 6 months.

In study 1, plasma from 203 peripheral blood samples from participants with primary GAD $(n=91)$ and healthy controls (HCs) $(n=112)$ were assayed for PACAP by radioimmunoassay. We excluded five samples (two HC and three GAD), so that participant numbers were $\mathrm{HC} n=110$ and GAD $n=88$. In study 2, a subset $n=87$ individuals with primary GAD with available DNA were genotyped for the PAC1R SNP.

\section{Procedure}

After informed consent, current and lifetime psychiatric diagnoses were assessed by a trained clinician using the Structured Clinical Interview for DSM-IV (SCID-IV) [49] or Mini International Neuropsychiatric Interview (MINI) [50]. Eligible participants completed clinician-administered and self-report clinical measures as well as peripheral blood draws for genetic and circulating biomarker analysis. All procedures were approved by the institutional review board at Partners Healthcare.

Blood processing. Blood samples were collected between 9:00 AM and 5:00 PM by trained research coordinators. Samples were centrifuged at $3500 \mathrm{rpm}$ for $20 \mathrm{~min}$ at $4{ }^{\circ} \mathrm{C}$. Plasma was extracted and stored at $-80^{\circ} \mathrm{C}$ until analysis.

Radioimmunoassay. PACAP38 radioimmunoassay was performed at the University of Vermont. Human plasma samples were concentrated on C18 Sep-Pak solid-phase cartridge minicolumns (Waters, Milford, MA) as described previously [51]. Briefly, the samples were recycled three times onto the minicolumns, washed with $0.1 \%$ trifluoroacetic acid (TFA) and eluted with $80 \%$ acetonitrile/0.1\% TFA. The eluates were dried under reduced pressure and resuspended in $0.1 \mathrm{M}$ sodium phosphate buffer (pH 7.4) containing $0.5 \%$ bovine serum albumin and $0.3 \mathrm{mg} / \mathrm{ml}$ phenylmethylsulfonylfluoride, immediately before assay. Peptide recovery from the Sep-Pak cartridges was $85 \%$. PACAP38 radioimmunoassay directed at the peptide a-amidated C-terminus $(1: 20,000)$ was performed using double antibody immunoprecipitation as previously described [52]. Each sample was assayed over a dilution range to ensure data interpolation. The assay midpoint was approximately $6.4-7.2 \mathrm{fmol}$ and detection limit from the linear range of the standard curve was $0.9 \mathrm{fmol}$. In analysis, we excluded five samples (three GAD, two HC) whose RIA PACAP levels were undetectable (readout on RIA was zero). Samples with peptide levels that were detectable but low confidence from values outside of the standard curve were collapsed to the $0.9 \mathrm{fmol}$ detection limit (study 1: 19 GAD, 8 HC; study 2: 2 genotype CC, 10 genotype CG, 7 genotype GG). 
Circulating PACAP peptide and PAC1R genotype as possible transdiagnostic... RA Ross et al.

Genotyping. Previously extracted DNA samples isolated from whole blood from 87/91 of the participants with primary GAD from study 1 were available for inclusion in study 2. Genotyping of rs2267735, an ADCYAP1R1 variant, was conducted via TaqMan assay.

Genotyping for rs2267735, located in an intronic region of the ADCYAP1R1 locus, was performed in the Ressler laboratory using a TaqMan R assay (Life Technologies Corporation, Carlsbad, CA) and run on the Applied Biosystems ${ }^{\mathrm{TM}}$ QuantStudio $^{\mathrm{TM}} 6$ Flex Real-Time PCR System. Individuals who conducted genotyping were blinded to participants' diagnoses.

\section{Measures}

The Hamilton Anxiety Rating Scale (HAM-A) [53, 54] was administered by trained clinicians to assess overall anxiety symptom severity, as well as subscale scores of somatic and psychological anxiety symptoms.The HAM-A is a well-validated measure of anxiety, and higher scores indicate increased clinical severity. The Pittsburgh Sleep Quality Index (PSQI) $[55,56]$ is a well-validated self-report scale that was administered to assess the severity of sleep disturbance and quality. A simplified version of the Traumatic Events Questionnaire (TEQ, modified for civilians) [57] was administered as a self-report questionnaire to assess whether participants had experienced one or more of ten types of traumatic events during their lifetime.

\section{Statistical methods}

For study 1, demographic and clinical symptom differences between patients with and without primary GAD were analyzed using Fisher's exact test for categorical outcomes and two-sample $t$ tests for continuous outcomes. To examine PACAP differences by diagnosis, we used a generalized linear model (GLM) with circulating PACAP as the dependent variable and diagnosis (GAD vs. HC), sex (male vs. female), and their interaction as predictors. PACAP values were log-transformed to better meet model assumptions of normality and homogeneity of variance. We tested the overall association between diagnosis and circulating PACAP using the main effect of diagnosis, and evaluated sexspecific effects using a priori contrasts of GAD vs. HC within each sex. In a follow-up model, we also included psychotropic medication use (yes/no), anti-inflammatory medication use (yes/ no), use of birth control medication (yes/no), and blood draw time (1-h intervals) as covariates to assess the sensitivity of our results to the inclusion of these additional potential confounding factors.

For study 2, sample characteristics between the three genotypes were examined using one-way ANOVAs for continuous and Fisher's exact test for categorical variables. The association of genotype with circulating PACAP (log-transformed), anxiety symptoms (HAM-A total and both subscales), and sleep symptoms were examined using separate GLMs for each outcome. Each GLM included the main effects of genotype and sex and the interaction of sex with genotype as predictors, as well as lifetime PTSD as a covariate. Based on our hypotheses that the CC genotype might be a risk genotype, we performed four tests: (1) difference between the CC genotype compared to the combination of the CG and GG genotypes overall; (2) same genotype comparison in males only; (3) same genotype comparison in females only and (4) difference between the genotype comparison in males vs. females (i.e., if there is a significant interaction between sex and the genotype effect). The $p$ values of the three anxiety severity symptom outcomes were adjusted for multiple comparisons using the false discovery rate adjustment.

Modeled means are presented as estimated marginal means (EMM) with 95\% confidence limits in the text and in Table 3, and effect sizes are Hedges' $g$ (i.e., effect sizes using weighted pooled standard deviations due to unequal group sizes) based on raw means. For circulating PACAP levels, which were analyzed after log-transformation, means are presented as estimated marginal geometric means (i.e., back-transformed EMMs) and effect sizes are based on raw means of log-transformed data.

\section{RESULTS}

Study 1: Circulating PACAP in patients with primary GAD and nonpsychiatric controls

Overall, participants were $35.6 \pm 13.5$ years old on average and were predominantly female $(67 \%)$, white $(76 \%)$, and non-Hispanic (92\%). Compared to participants without psychiatric disorders, patients with primary GAD in our sample were slightly younger, more likely to be white, more likely to have experienced one or more types of traumatic events during their lifetime, more likely to be taking antidepressant medication and benzodiazepines, and less likely to be taking anti-inflammatory medication (Table 1). The nonpsychiatric control group included a small minority of participants $(12 \%, n=12)$ who presented with mild or moderate levels of anxiety symptoms (Supplemental Fig. 1). Although there is no research available to be certain whether antidepressants, benzodiazepines or anti-inflammatory agents affect circulating PACAP, we adjusted for medication use differences in a follow-up analysis to the main model examining overall and sex-specific associations between GAD diagnosis and RIA PACAP.

Overall, there was no main effect of diagnosis (GAD vs. HC: $F$ $(1,194)=1.70, p=0.19, g=0.25)$ or sex (females vs. males: $F(1$, $194)=1.63, p=0.20, g=0.20$ ) on circulating PACAP. However, when examining diagnosis effects within sex subgroups, GAD (vs. $\mathrm{HC}$ ) was associated with lower concentrations of circulating PACAP in females $(13.6,95 \% \mathrm{Cl}$ : $[12.5,14.8]$ vs. $15.4[14.2,16.7] ; F(1$, $194)=4.10, p=0.04, g=0.33)$, but not in males $(15.4[13.4,17.6]$ vs. $15.6[14.0,17.4] ; F(1,194)=0.04, p=0.85, g=0.06)$ (Fig. 1). None of the covariates examined in a follow-up analysis were significantly associated with circulating PACAP (all $p>0.19$ ), and the addition of covariates in the main model did not alter the magnitude or significance of the effects observed in the simpler model that included only diagnosis, sex, and their interaction.

Study 2: PACAP levels, anxiety symptoms, and sleep disturbance by PAC1R genotype in patients with primary GAD

The subsample of adults with primary GAD included in study 2 $(n=87)$ were $32.6 \pm 11.2$ years old on average and were predominantly female $(70 \%)$, white $(90 \%)$, and non-Hispanic (94\%). Per prior reports in subjects with PTSD, CC genotype is thought to confer greater risk of symptoms [28]. We were unable to detect any differences in demographic variables, the prevalence of PTSD comorbidity, medication use, or clinical symptoms between the three genotypes (all $p>0.05$; Table 2). Similarly, we were unable to detect any main effects of genotype on circulating $\operatorname{PACAP}(p=0.11), \mathrm{HAM}-\mathrm{A}$ total anxiety severity $(p=0.30), \mathrm{HAM}-\mathrm{A}$ psychic symptom severity $(p=0.18)$, HAM-A somatic symptom severity $(p=0.62)$, or sleep disturbance $(p=0.43)$, and all specific contrasts of the risk genotype CC compared to the other two genotypes (CG and GG) for each outcome were not significant (all $p>0.20$; see Table 3).

However, specific contrasts of the sex difference in the CC vs. other genotypes comparison indicated that the CC genotype compared to the other genotypes was associated with higher somatic anxiety severity in females, but lower severity in males (adj $p=0.03$; Table 3, Fig. 2); we did not detect similar sex differences in HAM-A total scores $(p=0.37)$, HAM-A psychic severity scores $(p=0.92)$, sleep quality $(p=0.58$; Supplemental Fig. 2), nor in circulating PACAP levels ( $p=0.98$; Supplemental Fig. 3). Furthermore, lifetime diagnoses of comorbid PTSD were associated with a higher severity of sleep disturbance in patients with primary GAD ( $p=0.01$; see PSQI results in Table 3$)$, but were not significantly associated with any other outcome (Table 3 ). There is some indication that the CC genotype, compared to other genotypes, was associated with poorer sleep quality in women 
Table 1. Characteristics of participants with a primary diagnosis of generalized anxiety disorder $(n=88)$ and nonpsychiatric control participants $(n=110)$

\begin{tabular}{|c|c|c|c|}
\hline & Participants with GAD $(n=88)$ & Nonpsychiatric controls $(n=110)$ & Group difference \\
\hline Age, $y, M(S D)^{b}$ & $32.6(11.2)$ & $38.0(14.7)$ & $* *$ \\
\hline White, \% $(n)^{\mathrm{b}}$ & $90(79)$ & $65(70)$ & $* * *$ \\
\hline Black, \% $(n)^{\mathrm{b}}$ & $2(2)$ & $18(19)$ & *** \\
\hline Hispanic ethnicity, \% $(n)^{\mathrm{b}}$ & $6(5)$ & $10(11)$ & \\
\hline \multicolumn{4}{|l|}{ Clinical characteristics } \\
\hline HAM-A total scores, $M(S D)^{b}$ & $19.1(8.4)$ & $3.3(3.6)$ & $* * *$ \\
\hline Current PTSD, \% $(n)^{\mathrm{b}}$ & $7(6)$ & $0(0)$ & ** \\
\hline Lifetime PTSD, \% $(n)^{\mathrm{b}}$ & $14(12)$ & $0(0)$ & $* * *$ \\
\hline Mood stabilizers, \% (n) & $2(2)$ & $0(0)$ & \\
\hline Benzodiazepine, \% ( $n)$ & $20(18)$ & $5(6)$ & $* *$ \\
\hline Antipsychotics, \% (n) & $2(2)$ & $0(0)$ & \\
\hline \multicolumn{4}{|l|}{ Nonpsychotropic medication use } \\
\hline Any nonpsychotropic medication use, $\%(n)$ & $63(55)$ & $75(82)$ & \\
\hline Birth control (women only), \% ( $n$ ) & $23(14)$ & $24(17)$ & \\
\hline Anti-inflammatory, \% (n) & $45(40)$ & $65(71)$ & ** \\
\hline Aspirin, \% (n) & $24(21)$ & $25(27)$ & \\
\hline Statin, \% (n) & $6(5)$ & $5(5)$ & \\
\hline Other chronic medication, $\%(n)$ & $27(24)$ & $21(23)$ & \\
\hline
\end{tabular}

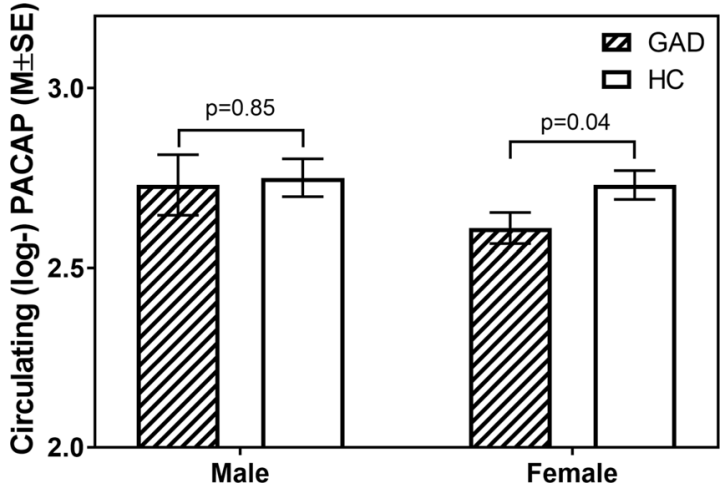

Fig. 1 Mean differences observed in circulating PACAP (logtransformed) by sex and diagnosis.

( $g=0.81$ ) but we were unable to detect a significant difference between genotype ( $p=0.06$, Table 3$)$.

\section{DISCUSSION}

PACAP is posited as an intriguing biomarker for anxiety disorders, with previously published utility for PTSD [28], and here we show it may likely be implicated in females with GAD as well. However, it is important to note that circulating PACAP levels alone may not be a useful biomarker for individuals with GAD and that additional, complementary measures of the peptidergic system may need to be combined. The differences in PACAP levels between the GAD and HC populations in the current study appear small, which challenge its utility as a diagnostic instrument, though more research is needed to understand if this may have other important clinical significance to identify potential treatment targets or optimal approaches for females with GAD.

Importantly, the results appear to suggest that PACAP levels may vary with expression level and genotype of the PACAP receptor, indicating that there may be a feedback mechanism by which the PAC1R influences circulating PACAP or vice versa [58-61]. The levels of PACAP and PAC1 receptor expression are tightly regulated for homeostasis and in some physiological paradigms, PACAP and the PAC1 receptors have a well-described reciprocal relationship. Marked increases in neural PACAP transcripts and proteins following axotomy and cyclophosphamide-induce cystitis, for example, were correlated with decreased PAC1 receptor transcript expression [62-65]. Hence we speculate that in patients with GAD, who have an elevated stress response even in the setting of minimal external stressors, the perceived experience of consistent stress may lead to alterations in this feedback including enhanced PAC1 receptor expression, which may impinge on peripheral PACAP signaling. Intriguingly, recent preliminary studies have 
Circulating PACAP peptide and PAC1R genotype as possible transdiagnostic... RA Ross et al.

Table 2. Characteristics of 87 participants with a primary diagnosis of generalized anxiety disorder, split by PAC1R SNP (rs2267735) genotype.

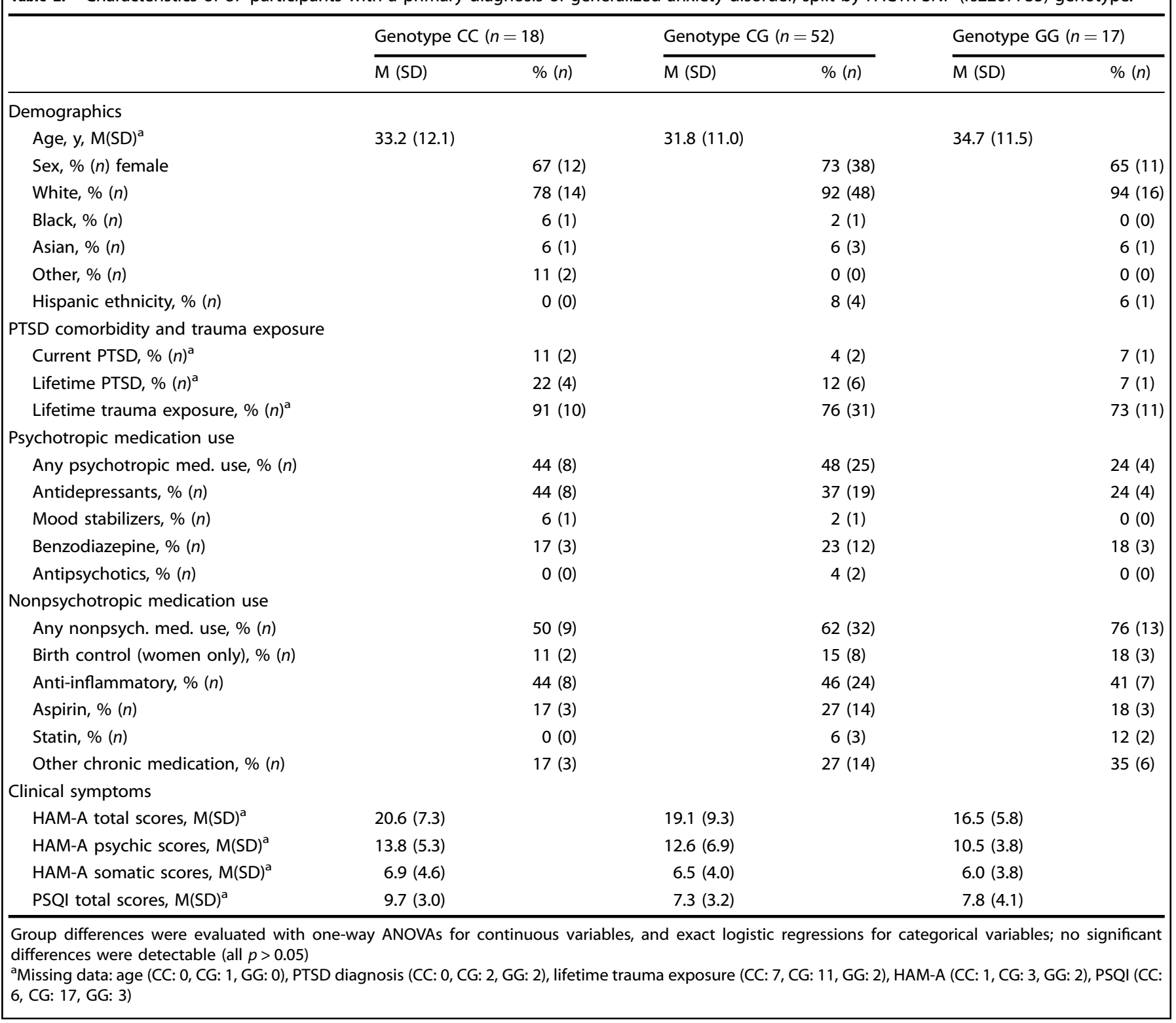

suggested that patients with moderate to high levels of anxiety have lower peripheral nerve PACAP content (May et al., unpublished observations). These patterns may be different from PTSD in which both central PACAP and PAC1 receptors appear elevated from chronic or severe traumatic stress [17]. Further investigation into the PACAPergic response to stress in the acute setting may be required to better elucidate the relationship between PAC1R and PACAP in patients with anxiety disorders and perceived high levels of stress.

The rs2267735 SNP (CC allele) has previously been shown to correlate with elevated PTSD symptom severity in females with PTSD [28], and here we find that the same genotype may have opposite associations with somatic anxiety severity in male and female patients with GAD (adj. $p=0.03$ for CC vs. other difference between males and females). Specifically, we show that females with GAD have lower circulating PACAP than healthy controls, and that females with the CC allele tend to have worse somatic symptoms of anxiety and insomnia than do other females. They also tend to have worse somatic symptoms of anxiety and report higher frequency and intensity of insomnia symptoms than males with the same genotype. Conversely, there is no difference in circulating PACAP between males with GAD and healthy controls, and males with the CC genotype of PAC1R have relatively mild somatic symptoms and insomnia when compared with males with either GG or CG genotype. This suggests an intriguing possibility that PACAP-related genotypic distinction may offer an important subcategorization of GAD of specific relevance for women, indicating potential similarities of gender specificity for PACAP across GAD and PTSD diagnosis. Further, it is possible that PACAP might be a transdiagnostic marker for somatic hyperarousal type symptoms in females regardless of diagnosis; future research is needed to replicate and extend our finding.

Overall, neural, genetic, and biological findings together suggest that PACAP levels and PAC1R genotype may be sexspecific biomarkers, linked to sensitized stress responding in females, but not males $[23,27,28,66]$. The PAC1R allele on which we focused has a known SNP in an estrogen response element, representing potential for a sex-based difference, including expression levels related to estrogen cycle [28]. This association with estrogen is of particular interest, given recent data 
Table 3. Estimated marginal means of specific effects examined in people with primary generalized anxiety disorder $(n=87$ ) in Study 2 .

\begin{tabular}{|c|c|c|c|c|}
\hline Outcome effect & Estimate $[95 \% \mathrm{Cl}]$ & $p$ & $\operatorname{adj} . p^{a}$ & $g^{c}$ \\
\hline \multicolumn{5}{|l|}{ Circulating PACAP [pM, log-transformed] } \\
\hline Genotype CC vs. other & $0.07[-0.04,0.18]$ & 0.20 & & 0.27 \\
\hline Female & $-0.07[-0.17,0.03]$ & 0.16 & & -0.33 \\
\hline Genotype CC vs. other in men & $0.07[-0.10,0.25]$ & 0.42 & & 0.30 \\
\hline Genotype CC vs. other in women & $0.07[-0.06,0.19]$ & 0.28 & & 0.24 \\
\hline Genotype CC vs. other difference ${ }^{b}$ & $0.00[-0.21,0.22]$ & 0.98 & & $\mathrm{n} / \mathrm{a}$ \\
\hline PTSD lifetime diagnosis & $-0.02[-0.26,0.23]$ & 0.88 & & -0.13 \\
\hline \multicolumn{5}{|c|}{ Total anxiety symptom severity (HAM-A total scores) } \\
\hline Genotype CC vs. other & $0.58[-2.02,3.18]$ & 0.66 & 0.66 & 0.26 \\
\hline Female & $-0.08[-2.47,2.30]$ & 0.94 & 0.94 & -0.11 \\
\hline Genotype CC vs. other in men & $-0.95[-5.29,3.39]$ & 0.66 & 0.66 & -0.25 \\
\hline Genotype CC vs. other in women & $2.11[-0.77,4.99]$ & 0.15 & 0.22 & 0.53 \\
\hline Genotype CC vs. other difference ${ }^{b}$ & $-3.06[-8.26,2.14]$ & 0.25 & 0.37 & $\mathrm{n} / \mathrm{a}$ \\
\hline PTSD lifetime diagnosis & $1.21[-4.45,6.88]$ & 0.67 & 0.80 & 0.18 \\
\hline \multicolumn{5}{|c|}{ Psychological anxiety symptom severity (HAM-A psychological scores) } \\
\hline Genotype CC vs. other & $1.00[-0.91,2.90]$ & 0.30 & 0.66 & 0.28 \\
\hline Female & $-0.89[-2.64,0.86]$ & 0.31 & 0.47 & -0.31 \\
\hline Genotype CC vs. other in men & $1.10[-2.08,4.27]$ & 0.49 & 0.66 & 0.18 \\
\hline Genotype CC vs. other in women & $0.90[-1.21,3.00]$ & 0.40 & 0.40 & 0.34 \\
\hline Genotype CC vs. other difference ${ }^{b}$ & $0.20[-3.61,4.01]$ & 0.92 & 0.92 & $\mathrm{n} / \mathrm{a}$ \\
\hline PTSD lifetime diagnosis & $0.89[-3.26,5.04]$ & 0.67 & 0.80 & 0.08 \\
\hline \multicolumn{5}{|c|}{ Somatic anxiety symptom severity (HAM-A somatic scores) } \\
\hline Genotype CC vs. other & $-0.41[-1.66,0.84]$ & 0.51 & 0.66 & 0.12 \\
\hline Female & $0.80[-0.35,1.94]$ & 0.17 & 0.47 & 0.25 \\
\hline Genotype CC vs. other in men & $-2.05[-4.12,0.03]$ & 0.05 & 0.16 & -1.00 \\
\hline Genotype CC vs. other in women & $1.22[-0.16,2.60]$ & 0.08 & 0.22 & 0.62 \\
\hline Genotype CC vs. other difference ${ }^{b}$ & $-3.27[-5.76,-0.77]$ & 0.01 & 0.03 & $\mathrm{n} / \mathrm{a}$ \\
\hline PTSD lifetime diagnosis & $0.35[-2.37,3.07]$ & 0.80 & 0.80 & 0.26 \\
\hline \multicolumn{5}{|l|}{ Sleep quality (PSQI global scores) } \\
\hline Genotype CC vs. other & $0.73[-0.62,2.08]$ & 0.28 & & 0.66 \\
\hline Female & $-0.29[-1.45,0.87]$ & 0.62 & & -0.15 \\
\hline Genotype CC vs. other in men & $0.35[-2.08,2.79]$ & 0.77 & & 0.07 \\
\hline Genotype CC vs. other in women & $1.11[-0.05,2.27]$ & 0.06 & & 0.81 \\
\hline Genotype CC vs. other difference ${ }^{b}$ & $-0.75[-3.45,1.94]$ & 0.58 & & $\mathrm{n} / \mathrm{a}$ \\
\hline PTSD lifetime diagnosis & $3.24[0.99,5.49]$ & 0.01 & & 1.07 \\
\hline \multicolumn{5}{|c|}{$\begin{array}{l}\text { Genotypes are PAC1R SNP (rs2267735) genotypes, and effects shown are based on specific contrasts between estimated marginal means estimated } \\
\text { single generalized linear model for each outcome } \\
\text { a adj. } p=\text { adjusted for false discovery rate among HAM-A total and subscales only } \\
{ }^{b} \text { Difference refers here to the sex difference in the CC vs. other (CG and GG) genotype effect (i.e., the interaction of gender and genotype effects) } \\
{ }^{c} \text { Hedges' } g \text { effect size of group differences based on raw means }\end{array}$} \\
\hline
\end{tabular}

supporting the role of estrogen and estrogen receptors in anxiety and PTSD [67-69]. In addition, in animals, central and peripheral PACAP (PACAP38, PACAP27) manipulations have been shown to alter sympathetic outflow, body temperature, breathing, heart rate, and headache [40, 44, 45, 70]. These alterations in sympathetic nervous system outflow due to PACAPergic dysfunction are relevant to GAD-type symptoms, particularly somatic symptoms, such as muscle tension, gastrointestinal upset, sweating, and increased arousal. Although no study to date has examined the specific relationship between somatic symptoms in anxiety disorders and PACAP, a few studies have supported that PACAP may induce somatic symptoms of anxiety in humans, including specifically headaches and altered breathing [71-75]. Thus, our sex-specific finding may reflect a difference in the underlying biology of the disorder related to presenting symptoms, specifically due to dysfunction in the PACAPergic circuitry of the sympathetic nervous system and may pose a unique treatment target for a subset of patients with primarily somatic experience of anxiety.

Limitations of our study include a relatively low sample size for the number of variables, reducing our capacity to detect statistically significant specific associations. We were particularly underpowered to detect differences in males, because the CC allele was present in far fewer male than female samples. This again indicates a potential biological difference underlying sexvariance in presentation and etiology of anxiety disorders. Furthermore, serum was from samples collected from participants at the time of their presentation to the research clinic, without consideration of time of day, metabolic status, or estrogen cycling in females. Given PACAP involvement in each of these 


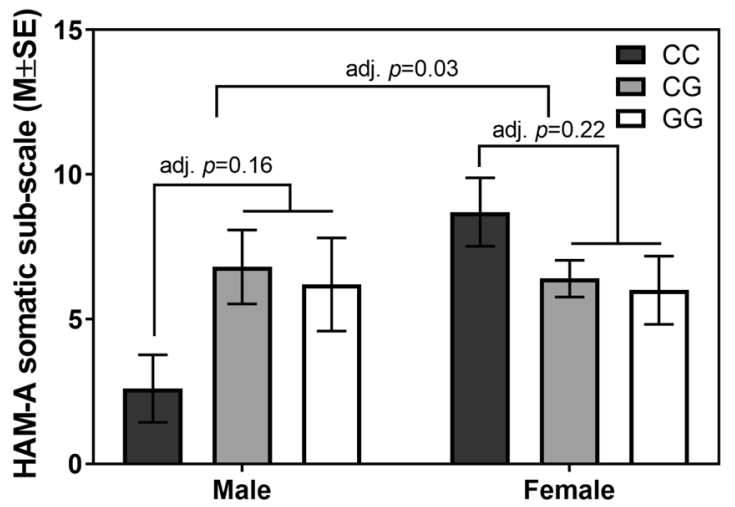

Fig. 2 Means of somatic anxiety symptom severity (HAM-A somatic) by sex and PAC1R SNP (rs2267735) genotype.

homeostatic processes $[8,76,77]$, it is prudent to consider these potential variables in future analysis. Finally, there was a high degree of reported lifetime trauma exposure for both the $\mathrm{HC}$ and the GAD samples, which may be relevant to circulating PACAP levels and to the interaction of PACAP with the PAC1R over time. Future studies should include assessments of trauma exposure, type, and intensity to improve our understanding of how gene and environment (i.e., trauma exposure) interactions may impact circulating PACAP levels in patients with anxiety disorders. Larger sample sizes (i.e., $n>150$ or greater) would allow examination of whether PACAP may be mediating the association between genotype and symptom type, and future studies could also investigate the impact of estrogen levels on PACAP. These initial suggestive data support that further study, with larger sample sizes, controlled serum collection, and deeper examination of acute reactivity and chronic somatic hyperarousal, is needed. With additional evidence and elucidation of mechanism, PACAP and its associated functional markers may be useful as a transdiagnostic biomarker for clinical diagnosis and/or treatment of anxiety and stress-related disorders.

\section{CONCLUSION}

Here we show that while circulating PACAP is not associated with a main effect of GAD diagnosis, it may be associated with GAD in females only. Additionally among patients with GAD, the risk genotype identified in the PTSD literature ( $r$ 2267735, CC genotype) is associated with anxiety symptom severity for females. Specifically, the association between the risk genotype, circulating PACAP, and somatic anxiety severity is stronger among females than males. This indicates a potential underlying biological etiology for sex differences in stress-related anxiety disorders that warrants further study.

\section{FUNDING AND DISCLOSURE}

This work was funded in part by a generous grant from the Highland Street Foundation (NMS). Support was also received from NIH (MH096764 and MH115874 (KJR)). RAR is supported in part by funding from the NIH through grants from the NIMH (R25 MH094612), NHLBI (5T32HL007374-36), NCATS (UL1 TR001102), and NIDDK (DK118201-01A1).

RAR has no competing interests in relation to the manuscript content and no financial disclosures to report. KJR holds patents for use of D-cycloserine to enhance fear extinction, as well as for targeting PAC1, tachykinin 2, and angiotensin to improve the extinction of fear. RAR is also on scientific advisory boards for Janssen and Verily, and has received research funding from Brainsway, Takeda and Alkermes. NMS has no competing interests in relation to the manuscript content. In the past 36 months, NMS has received research support from the American Foundation for Suicide Prevention, Department of Defense, PCORI, Highland Street Foundation, $\mathrm{NIH}$, and Janssen. NMS reports consulting for Axovant Sciences, Springworks, Praxis Therapeutics, Aptinyx, Genomind, Wolters Kluwer (royalty) and spousal equity in G1 Therapeutics. The remaining authors have no additional conflicts of interest or disclosures to report.

\section{AUTHOR CONTRIBUTIONS}

RAR, KJR, and NMS conceived the present study design and aims. RAR, SNH, EBO, and PLR acquired, managed, and cleaned study data. VM conducted radioimmunoassays and assisted in the interpretation of findings. KJR coordinated genotyping and consulted on the interpretation of data. SSH and RAR devised the data analytic plan and conducted all analyses. RAR, SNH, and SSH drafted the manuscript, and KJR, VM, and NMS provided revisions. All authors reviewed and approved of the final manuscript. We appreciate our reviewers thoughtful input and recommendations for future research directions in the revision process.

\section{ADDITIONAL INFORMATION}

Supplementary Information accompanies this paper at (https://doi.org/10.1038/ s41386-020-0604-4).

Publisher's note Springer Nature remains neutral with regard to jurisdictional claims in published maps and institutional affiliations.

\section{REFERENCES}

1. Stroth N, Holighaus $Y$, Ait-Ali D, Eiden LE, Ait-Ali D, Eiden LE. PACAP: a master regulator of neuroendocrine stress circuits and the cellular stress response. Ann N Y Acad Sci. 2011;1220:49-59.

2. Hashimoto $H$, Shintani N, Tanida M, Hayata A, Hashimoto R, Baba A. PACAP is implicated in the stress axes. Curr Pharm Des. 2011;17:985-9.

3. Masuo $Y$, Suzuki N, Matsumoto $H$, Tokito $F$, Matsumoto $Y$, Tsuda $M$, et al. Regional distribution of pituitary adenylate cyclase activating polypeptide (PACAP) in the rat central nervous system as determined by sandwich-enzyme immunoassay. Brain Res. 1993;602:57-63.

4. Ghatei MA, Takahashi K, Suzuki Y, Gardiner J, Jones PM, Bloom SR. Distribution, molecular characterization of pituitary adenylate cyclase-activating polypeptide and its precursor encoding messenger RNA in human and rat tissues. J Endocrinol. 1993;136:159-66.

5. Palkovits M, Somogyvári-Vigh A, Arimura A. Concentrations of pituitary adenylate cyclase activating polypeptide (PACAP) in human brain nuclei. Brain Res. 1995;699:116-20.

6. Arimura A, Somogyvári-Vigh A, Miyata A, Mizuno K, Coy DH, Kitada C. Tissue distribution of PACAP as determined by RIA: highly abundant in the rat brain and testes. Endocrinology. 1991;129:2787-9.

7. Moller K, Sundler F. Expression of pituitary adenylate cyclase activating peptide (PACAP) and PACAP type I receptors in the rat adrenal medulla. Regul Pept. 1996;63:129-39.

8. Chen D, Buchanan GF, Ding JM, Hannibal J, Gillette MU. Pituitary adenylyl cyclaseactivating peptide: a pivotal modulator of glutamatergic regulation of the suprachiasmatic circadian clock. Proc Natl Acad Sci USA. 1999;96:13468-73.

9. Tsukiyama N, Saida $Y$, Kakuda M, Shintani N, Hayata A, Morita $Y$, et al. PACAP centrally mediates emotional stress-induced corticosterone responses in mice. Stress. 2011;14:368-75.

10. Dore $R$, lemolo A, Smith KL, Wang $X$, Cottone $P$, Sabino V. CRF mediates the anxiogenic and anti-rewarding, but not the anorectic effects of PACAP. Neuropsychopharmacology. 2013;38:2160-9.

11. Lehmann ML, Mustafa T, Eiden AM, Herkenham M, Eiden LE. PACAP-deficient mice show attenuated corticosterone secretion and fail to develop depressive behavior during chronic social defeat stress. Psychoneuroendocrinology. 2013;38:702-15.

12. Hattori S, Takao K, Tanda K, Toyama K, Shintani N, Baba A, et al. Comprehensive behavioral analysis of pituitary adenylate cyclase-activating polypeptide (PACAP) knockout mice. Front Behav Neurosci. 2012;6:1-18.

13. Otto C, Martin M, Paul Wolfer D, Lipp HP, Maldonado R, Schütz G. Altered emotional behavior in PACAP-type-I-receptor-deficient mice. Mol Brain Res. 2001;92:78-84.

14. King SB, Lezak KR, O'Reilly M, Toufexis DJ, Falls WA, Braas K, et al. The effects of prior stress on anxiety-like responding to intra-BNST pituitary adenylate cyclase activating polypeptide in male and female rats. Neuropsychopharmacology. 2017;42:1679-87. 
15. Lezak KR, Roelke E, Harris OM, Choi I, Edwards S, Gick N, et al. Pituitary adenylate cyclase-activating polypeptide (PACAP) in the bed nucleus of the stria terminalis (BNST) increases corticosterone in male and female rats. Psychoneuroendocrinology. 2014;45:11-20.

16. lemolo A, Seiglie M, Blasio A, Cottone $P$, Sabino V. Pituitary adenylate cyclaseactivating polypeptide (PACAP) in the central nucleus of the amygdala induces anxiety via melanocortin receptors. Psychopharmacology (Berlin). 2016;233: 3269-77.

17. Hammack SE, Cheung J, Rhodes KM, Schutz KC, Falls WA, Braas KM, et al. Chronic stress increases pituitary adenylate cyclase-activating peptide (PACAP) and brainderived neurotrophic factor (BDNF) mRNA expression in the bed nucleus of the stria terminalis (BNST): roles for PACAP in anxiety-like behavior. Psychoneuroendocrinology. 2009;34:833-43.

18. Lezak KR, Roman CW, Braas KM, Schutz KC, Falls WA, Schulkin J, et al. Regulation of bed nucleus of the stria terminalis PACAP expression by stress and corticosterone. J Mol Neurosci. 2014;54:477-84.

19. Meloni EG, Venkataraman A, Donahue RJ, Carlezon WA. Bi-directional effects of pituitary adenylate cyclase-activating polypeptide (PACAP) on fear-related behavior and c-Fos expression after fear conditioning in rats. Psychoneuroendocrinology. 2016;64:12-21.

20. Shea A, Walsh C, MacMillan H, Steiner M. Child maltreatment and HPA axis dysregulation: relationship to major depressive disorder and post traumatic stress disorder in females. Psychoneuroendocrinology. 2005;30:162-78.

21. de Kloet CS, Vermetten E, Geuze E, Kavelaars A, Heijnen CJ, Westenberg HGM. Assessment of HPA-axis function in posttraumatic stress disorder: pharmacological and non-pharmacological challenge tests, a review. J Psychiatr Res. 2006;40:550-67.

22. Blechert J, Michael T, Vriends $\mathrm{N}$, Margraf J, Wilhelm FH. Fear conditioning in posttraumatic stress disorder: evidence for delayed extinction of autonomic, experiential, and behavioural responses. Behav Res Ther. 2007;45:2019-33.

23. Mercer KB, Dias B, Shafer D, Maddox SA, Mulle JG, Hu P, et al. Functional evaluation of a PTSD-associated genetic variant: estradiol regulation and ADCYAP1R1. Transl Psychiatry. 2016;6:1-7.

24. Lowe SR, Pothen J, Quinn JW, Rundle A, Bradley B, Galea S, et al. Gene-by-socialenvironment interaction (GXSE) between ADCYAP1R1 genotype and neighborhood crime predicts major depression symptoms in trauma-exposed women. J Affect Disord. 2015;187:147-50.

25. Uddin M, Chang S-C, Zhang C, Ressler K, Mercer KB, Galea S, et al. Adcyap1r1 genotype, posttraumatic stress disorder, and depression among women exposed to childhood maltreatment. Depress Anxiety. 2013;30:251-8.

26. Chang SC, Xie P, Anton RF, De Vivo I, Farrer LA, Kranzler HR, et al. No association between ADCYAP1R1 and post-traumatic stress disorder in two independent samples. Mol Psychiatry. 2012;17:239-41.

27. Almli LM, Mercer KB, Kerley K, Feng H, Bradley B, Conneely KN, et al. ADCYAP1R1 genotype associates with post-traumatic stress symptoms in highly traumatized African-American females. Am J Med Genet Part B Neuropsychiatr Genet. 2013;162:262-72.

28. Ressler KJ, Mercer KB, Bradley B, Jovanovic T, Mahan A, Kerley K, et al. Posttraumatic stress disorder is associated with PACAP and the PAC1 receptor. Nature. 2011;470:492

29. Faravelli C, Sauro C Lo, Lelli L, Pietrini F, Lazzeretti L, Godini L, et al. The role of life events and HPA axis in anxiety disorders: a review. Curr Pharm Des. 2012;18:0-0.

30. Lader, M. Generalized anxiety disorder. In: Encyclopedia of psychopharmacology. Berlin Heidelberg: Springer; 2015, p. 699-702. https://doi.org/10.1007/978-3-64236172-2_317

31. American Psychiatric Association. Diagnostic and statistical manual of mental disorders (DSM-5 ${ }^{\circledR}$ ). American Psychiatric Pub; Washington, DC, 2013.

32. Kessler RC, Petukhova M, Sampson NA, Zaslavsky AM, Wittchen H-U. Twelvemonth and lifetime prevalence and lifetime morbid risk of anxiety and mood disorders in the United States. Int J Methods Psychiatr Res. 2012;21:169-84.

33. Amstadter AB, Koenen KC, Ruggiero KJ, Acierno R, Galea S, Kilpatrick DG, et al. NPY moderates the relation between hurricane exposure and generalized anxiety disorder in an epidemiologic sample of hurricane-exposed adults. Depress Anxiety. 2010;27:270-5.

34. Cooper AJ, Narasimhan S, Rickels K, Lohoff FW. Genetic polymorphisms in the PACAP and PAC1 receptor genes and treatment response to venlafaxine XR in generalized anxiety disorder. Psychiatry Res. 2013;210:1299-1300.

35. Murck H, Steiger A, Frieboes RM, Antonijevic IA. Pituitary adenylate cyclaseactivating peptide affects homeostatic sleep regulation in healthy young men. Am J Physiol Metab. 2007:292:E853-7.

36. Tsypes A, Aldao A, Mennin DS. Emotion dysregulation and sleep difficulties in generalized anxiety disorder. J Anxiety Disord. 2013;27:197-203.

37. Hannibal J, Ding JM, Chen D, Fahrenkrug J, Larsen PJ, Gillette MU, et al. Pituitary adenylate cyclase-activating peptide (PACAP) in the retinohypothalamic tract: a potential daytime regulator of the biological clock. J Neurosci. 1997;17:2637-44.
38. Cagampang FRA, Piggins HD, Sheward WJ, Harmar AJ, Coen CW. Circadian changes in PACAP type 1 (PAC1) receptor mRNA in the rat suprachiasmatic and supraoptic nuclei. Brain Res. 1998;813:218-22.

39. Harrington ME, Hoque S, Hall A, Golombek D, Biello S. Pituitary adenylate cyclase activating peptide phase shifts circadian rhythms in a manner similar to light. J Neurosci. 1999;19:6637-42.

40. Inglott MA, Farnham MMJ, Pilowsky PM. Intrathecal PACAP-38 causes prolonged widespread sympathoexcitation via a spinally mediated mechanism and increases in basal metabolic rate in anesthetized rat. Am J Physiol Hear Circ Physiol. 2011;300:2300-7.

41. Farnham MMJ, Inglott MA, Pilowsky PM. Intrathecal PACAP-38 causes increases in sympathetic nerve activity and heart rate but not blood pressure in the spontaneously hypertensive rat. Am J Physiol Circ Physiol. 2010;300:H214-22.

42. Farnham MMJ, Li Q, Goodchild AK, Pilowsky PM. PACAP is expressed in sympathoexcitatory bulbospinal C1 neurons of the brain stem and increases sympathetic nerve activity in vivo. Am J Physiol Integr Comp Physiol. 2008;294:R1304-11.

43. Lindén A, Cardell L-O, Yoshihara $S$, Stjärne $P$, Nadel JA. PACAP 1-38 as an inhaled bronchodilator in guinea pigs in vivo. Peptides. 1998;19:93-8.

44. Wilson RJA, Cummings KJ. Pituitary adenylate cyclase-activating polypeptide is vital for neonatal survival and the neuronal control of breathing. Respir Physiol Neurobiol. 2008;164:168-78.

45. Syed AU, Koide M, Braas KM, May V, Wellman GC. Pituitary adenylate cyclaseactivating polypeptide (PACAP) potently dilates middle meningeal arteries: implications for migraine. J Mol Neurosci. 2012;48:574-83.

46. Hoge EA, Marques L, Wechsler RS, Lasky AK, Delong HR, Jacoby RJ, et al. The role of anxiety sensitivity in sleep disturbance in panic disorder. J Anxiety Disord. 2011;25:536-8

47. Baker AW, Keshaviah A, Goetter EM, Bui E, Swee M, Rosencrans PL, et al. Examining the role of anxiety sensitivity in sleep dysfunction across anxiety disorders. Behav Sleep Med. 2017;15:216-27.

48. Baker AW, Goetter EM, Bui E, Shah R, Charney ME, Mauro C, et al. The influence of anxiety sensitivity on a wish to die in complicated grief. J Nerv Ment Dis. 2016;204:314-6.

49. First MB, Spitzer RL, Gibbon M, Williams, JBW. Structured clinical interview for DSM-IV axis I disorders. New York State Psychiatric Institute; New York, NY, 1995.

50. Sheehan DV, Lecrubier Y, Sheehan KH, Amorim P, Janavs J, Weiller E, et al. The Mini-International Neuropsychiatric Interview (M.I.N.I.): the development and validation of a structured diagnostic psychiatric interview for DSM-IV and ICD-10. J Clin Psychiatry. 1998;59(Suppl 2):22-33. quiz 34-57

51. Brandenburg CA, May V, Braas KM. Identification of endogenous sympathetic neuron pituitary adenylate cyclase-activating polypeptide (PACAP): depolarization regulates production and secretion through induction of multiple propeptide transcripts. J Neurosci. 1997;17:4045-55.

52. Girard BA, Lelievre V, Braas KM, Razinia T, Vizzard MA, loffe $Y$, et al. Noncompensation in peptide/receptor gene expression and distinct behavioral phenotypes in VIP- and PACAP-deficient mice. J Neurochem. 2006:99:499-513.

53. Hamilton M. The assessments of anxiety rating. Br J Med Psychol. 1959;32:50-5.

54. Shear MK, Vander Bilt J, Rucci P, Endicott J, Lydiard B, Otto MW, et al. Reliability and validity of a structured interview guide for the Hamilton Anxiety Rating Scale (SIGH-A). Depress Anxiety. 2001;13:166-78.

55. Buysse DJ, Reynolds CF, Monk TH, Berman SR, Kupfer DJ. The Pittsburgh sleep quality index: a new instrument for psychiatric practice and research. Psychiatry Res. 1989;28:193-213.

56. Carpenter JS, Andrykowski MA. Psychometric evaluation of the pittsburgh sleep quality index. J Psychosom Res. 1998;45:5-13.

57. Vrana S, Lauterbach D. Prevalence of traumatic events and post-traumatic psychological symptoms in a nonclinical sample of college students. J Trauma Stress. 1994;7:289-302.

58. Cline DL, Short LI, Forster MAM, Gray SL. Adipose tissue expression of PACAP, VIP, and their receptors in response to cold stress. J Mol Neurosci. 2018. https://doi. org/10.1007/s12031-018-1099-x

59. Daniel PB, Kieffer TJ, Leech CA, Habener JF. Novel alternatively spliced exon in the extracellular ligand-binding domain of the pituitary adenylate cyclase-activating polypeptide (PACAP) type 1 receptor (PAC1R) selectively increases ligand affinity and alters signal transduction coupling during sper. J Biol Chem. 2001;276:12938-44.

60. Stroth N, Liu Y, Aguilera G, Eiden LE. Pituitary adenylate cyclase-activating polypeptide controls stimulus-transcription coupling in the hypothalamicpituitary-adrenal axis to mediate sustained hormone secretion during stress. J Neuroendocrinol. 2011;23:944-55.

61. Kanasaki H, Mutiara S, Oride A, Purwana IN, Miyazaki K. Pulse frequencydependent gonadotropin gene expression by adenylate cyclase-activating polypeptide 1 in perifused mouse pituitary gonadotroph LbetaT2 cells1. Biol Reprod. 2009;81:465-72.

62. Moller K, Reimer M, Ekblad E, Hannibal J, Fahrenkrug J, Kanje M, et al. The effects of axotomy and preganglionic denervation on the expression of 
pituitary adenylate cyclase activating peptide (PACAP), galanin and PACAP type 1 receptors in the rat superior cervical ganglion. Brain Res. 1997;775: $166-82$.

63. Zhou X, Rodriguez WI, Casillas RA, Ma V, Tam J, Hu Z, et al. Axotomy-induced changes in pituitary adenylate cyclase activating polypeptide (PACAP) and PACAP receptor gene expression in the adult rat facial motor nucleus. J Neurosci Res. 1999;57:953-61.

64. Jongsma H, Danielsen N, Sundler F, Kanje M. Alteration of PACAP distribution and PACAP receptor binding in the rat sensory nervous system following sciatic nerve transection. Brain Res. 2000;853:186-96.

65. Girard BM, Tompkins JD, Parsons RL, May V, Vizzard MA. Effects of CYP-induced cystitis on PACAP/VIP and receptor expression in micturition pathways and bladder function in mice with overexpression of NGF in urothelium. J Mol Neurosci. 2012;48:730-43.

66. Pohlack ST, Nees F, Ruttorf M, Cacciaglia R, Winkelmann T, Schad LR, et al. Neural mechanism of a sex-specific risk variant for posttraumatic stress disorder in the type I eeceptor of the pituitary adenylate cyclase activating polypeptide. Biol Psychiatry. 2015;78:840-7.

67. Maeng LY, Milad MR. Sex differences in anxiety disorders: interactions between fear, stress, and gonadal hormones. Horm Behav. 2015;76:106-17.

68. Fındıklı E, Camkurt MA, Karaaslan MF, Kurutas EB, Altun H, İzci F, et al. Serum levels of $\mathrm{G}$ protein-coupled estrogen receptor 1 (GPER1) in drug-naive patients with generalized anxiety disorder. Psychiatry Res. 2016;244:312-6.

69. Ryan J, Scali J, Carrière I, Scarabin P-Y, Ritchie K, Ancelin M-L. Estrogen receptor gene variants are associated with anxiety disorders in older women. Psychoneuroendocrinology. 2011;36:1582-6.
70. Masuo Y, Noguchi J, Morita S, Matsumoto Y. Effects of intracerebroventricular administration of pituitary adenylate cyclase-activating polypeptide (PACAP) on the motor activity and reserpine-induced hypothermia in murines. Brain Res. 1995;700:219-26.

71. Vécsei L, Tuka B, Tajti J. Role of PACAP in migraine headaches. Brain 2014;137:650-1.

72. Lindén A, Hansson L, Andersson A, Palmqvist M, Arvidsson P, Löfdahl C-G, et al. Bronchodilation by an inhaled VPAC(2) receptor agonist in patients with stable asthma. Thorax. 2003;58:217-21.

73. Amin FM, Sohail Asghar M, Guo S, Hougaard A, Hansen AE, Schytz HW, et al. Headache and prolonged dilatation of the middle meningeal artery by PACAP38 in healthy volunteers. Cephalalgia. 2012. https://doi.org/10.1177/ 0333102411431333

74. Yoshihara S, Yamada Y, Abe T, Kashimoto K, Lindén A, Arisaka O. Long-lasting smooth-muscle relaxation by a novel PACAP analogue in human bronchi. Regul Pept. 2004;123:161-5.

75. Kinhult J, Andersson JA, Uddman R, Stjärne P, Cardell LO. Pituitary adenylate cyclase-activating peptide 38 a potent endogenously produced dilator of human airways. Eur Respir J. 2000;15:243-7.

76. Resch JM, Boisvert JP, Hourigan AE, Mueller CR, Yi SS, Choi S. Stimulation of the hypothalamic ventromedial nuclei by pituitary adenylate cyclase-activating polypeptide induces hypophagia and thermogenesis. Am J Physiol Integr Comp Physiol. 2011;301:R1625-34.

77. Ross RA, Leon S, Madara JC, Schafer D, Fergani C, Maguire CA, et al. PACAP neurons in the ventral premammillary nucleus regulate reproductive function in the female mouse. Elife. 2018;7:e35960. 